\title{
Erste Hilfe für Lebensmittel - Konsumentenorientierte Vermeidungsmaßnahmen entwickeln, umsetzen und evaluieren
}

\author{
Sandra Schwödt · Gudrun Obersteiner
}

Online publiziert: 18. März 2019

(c) Der/die Autor(en) 2019

Zusammenfassung Im Rahmen einer Pilotaktion des EU-Projekts STREFOWA wurde am Institut für Abfallwirtschaft (Universität für Bodenkultur Wien) die Erste-Hilfe-Box für Lebensmittel entwickelt. Die Inhalte der Box basieren auf einer von April bis Mai 2017 in Österreich durchgeführten Online-Haushaltsumfrage mit über 2159 TeilnehmerInnen. Die Inhalte wurden an die Ergebnisse der Umfrage und somit an die Bedürfnisse der KonsumentInnen betreffend Wissen, Ausbau genutzter Vermeidungsmaßnahmen sowie Einsatz neuer Hilfsmittel angepasst. Bei der Verteilung von 2000 Boxen wurde auch darauf Rücksicht genommen, auf welche Art und Weise die KonsumentInnen bevorzugt kontaktiert werden möchten. In weiterer Folge wurden die Materialien mittels Befragung evaluiert, um Rückschlüsse ziehen zu können, inwiefern die Materialien den KonsumentInnen weiterhelfen konnten und um von diesen Inputs für die Überarbeitung der Materialien und zukünftige Abfallvermeidungsmaßnahmen zu erhalten.

\section{First Aid for Food - Develop, implement and evaluate consumer-oriented food waste prevention measures}

Abstract As part of a pilot action of the EU project STREFOWA, the Institute of Waste Management (BOKU University) developed the so called "First Aid Box for Food". The contents of the box are based on an online household survey conducted in Austria from April to May 2017 with more than 2159 participants. The contents were adapted to

DI S. Schwödt $(\bowtie) \cdot$ DI G. Obersteiner Department Wasser - Atmosphäre Umwelt, Institut für Abfallwirtschaft, Universität für Bodenkultur Wien, Muthgasse 107, 1190 Wien, Österreich sandra.schwoedt@boku.ac.at the results of the survey and thus to the needs of the consumers, concerning knowledge, enhancing already used food waste prevention measures as well as the use of new aids. The preferences of the consumers concerning type and frequency of contacting have also been taken into account. 2000 boxes have been handed out, subsequently, the materials were evaluated by means of questioning in order to be able to draw conclusions on how the materials could help the consumers and to receive from them more inputs for the revision of the materials and future waste prevention measures.

\section{Einleitung}

Das 12. Ziel für eine nachhaltige Entwicklung (Sustainable Developement Goals, SDGs) der Vereinten Nationen setzt sich mit nachhaltigen Konsumund Produktionsweisen auseinander. Der Unterpunkt 12.3 sieht eine Verringerung der Lebensmittelabfälle pro Kopf um $50 \%$ auf Ebene des Handels bis hin $\mathrm{zu}$ jener der KonsumentInnen vor. Die vorliegende Arbeit diente dazu, maßgeschneiderte Vermeidungsmaßnahmen für KonsumentInnen zu entwickeln, auf deren Ebene das größte Vermeidungspotenzial in Europa (Stenmarck et al. 2016) besteht. Doch nachhaltige Wertschöpfungsketten sowie die Vermeidung von Lebensmittelabfällen sind auch für die Erreichung anderer SDGs wie 1 (Zero Poverty), 2 (Zero Hunger) und 3 (Good Health and WellBeing) unerlässlich.

Die Umsetzung von Lebensmittelabfallvermeidungsmaßnahmen in Haushalten bzw. das Erreichen von Haushalten, sodass eine tatsächliche Verhaltensänderung eintritt, hat sich bislang als schwierig erwiesen, obwohl es nicht an Initiativen mangelt. Daher ist es wichtig, mehr darüber in Erfahrung zu bringen, was KonsumentInnen dazu motiviert bzw. daran hindert, Le- bensmittelabfälle $\mathrm{zu}$ vermeiden, um anschließend auf die Bedürfnisse der KonsumentInnen maßgeschneiderte Vermeidungsmaßnahmen entwickeln zu können.

Die Gründe für das Aufkommen von Lebensmittelabfällen in Haushalten sind vielfältig. Sie können sowohl mit der persönlichen Lebenssituation und sozio-demografischen Einflussfaktoren in Zusammenhang stehen, als auch mit Einstellungen, Gewohnheiten und Wissen in Verbindung gebracht werden. Drei wichtige Anhaltspunkte, um einschätzen zu können, inwiefern KonsumentInnen gewillt sind, Lebensmittelabfälle $\mathrm{zu}$ vermeiden, stellen deren Sensibilität gegenüber Lebensmittelabfällen, deren Vermeidungsanstrengungen sowie die Motivation dar, das eigene Verhalten $\mathrm{zu}$ ändern.

Wichtig erscheint außerdem nicht nur, eine Fülle an unterschiedlichen Maßnahmen zu entwickeln, sondern diese anschließend auch gründlich zu evaluieren, um in Zukunft daraus lernen und darauf aufbauen zu können. Dies wurde bisher in Österreich nur sehr selten bei für KonsumentInnen entwickelten Maßnahmen zur Lebensmittelabfallvermeidung durchgeführt.

Im Rahmen des Projektes STREFOWA wurde im Frühjahr 2017 vom Institut für Abfallwirtschaft eine Haushaltsumfrage durchgeführt (Obersteiner und Schwödt 2017), deren Ziel es unter anderem war, Chancen und Hindernisse für die Entwicklung von Lebensmittelabfallvermeidungsmaßnahmen $\mathrm{zu}$ identifizieren. Erstmals konnten dabei auch verschiedene KonsumentInnengruppen identifiziert werden. Basierend auf diesen Erkenntnissen wurde eine Erste-Hilfe-Box für Lebensmittel entwickelt und getestet, welche im vorliegenden Artikel näher vorgestellt wird. 


\section{Bedürfnisse der \\ KonsumentInnen}

Um maßgeschneiderte Maßnahmen für KonsumentInnen entwickeln zu können, ist es in erster Linie wichtig, deren Präferenzen und Wünsche hinsichtlich der Kommunikation von Lebensmittelabfallvermeidungsmöglichkeiten zu erheben, aber auch ihren Wissenstand zu potenziellen Auswirkungen aber auch Vermeidungsmöglichkeiten zu kennen, um in weiterer Folge besser auf die individuellen Bedürfnisse eingehen zu können.

Folgende Daten basieren auf der vom Institut für Abfallwirtschaft in Österreich durchgeführten Haushaltsumfrage. Dabei wurden 2159 TeilnehmerInnen $\mathrm{zu}$ ihrer Einstellung, ihrem Wissen sowie momentan in den Haushalten durchgeführten Vermeidungsmaßnahmen bzw. zum Lebensmittelabfallaufkommen befragt.

\subsection{Präferenzen bei der Kontaktaufnahme}

Obwohl unterschiedliche KonsumentInnengruppen identifiziert werden konnten, unterscheiden sich diese kaum bezüglich der gewünschten Häufigkeit der Kontaktaufnahme, der gewünschten Medien zur Kontaktaufnahme bzw. auch der Art der gewünschten Informationen.

\subsubsection{Häufigkeit der Kontaktaufnahme}

Betreffend die Häufigkeit der gewünschten Informationen zeigt sich ein einheitliches Bild. $41 \%$ der Befragten ziehen es vor, nur einmalig kontaktiert $\mathrm{zu}$ werden, damit sie wissen, wo die Materialien bei Bedarf abrufbar sind. Weitere $37 \%$ können sich vorstellen, in größeren Abständen (max. $1 \times$ pro Monat) kontaktiert zu werden. Mehr als einmal pro Monat kontaktiert zu werden, können sich nur $14 \%$ der Befragten vorstellen, während $8 \%$ gar nicht kontaktiert werden möchten.

\subsubsection{Gewünschte Wege zur Kontaktaufnahme}

Ein interessanter Aspekt ist, dass sich ein Großteil der befragten KonsumentInnen (ca. $74 \%$ ) bei vorgegebenen Antwortmöglichkeiten Informationen über Lebensmittelabfallvermeidungsmaßnahmen bzw. auch Tipps zur richtigen Lagerung direkt auf Produktverpackun- gen wünscht, ca. $46 \%$ der Befragten wünschen sich, dass entsprechende Informationen im Supermarkt aufliegen. Die Produktion bzw. der Einzelhandel scheinen zur Informationsvermittlung aus Sicht der KonsumentInnen eine wichtige Rolle $\mathrm{zu}$ spielen und sollten in Lebensmittelvermeidungsmaßnahmen bzw. Kampagnen miteinbezogen werden.

Nicht zu unterschätzen ist auch die Reichweite bzw. der Einfluss sozialer Medien, immerhin 30\% der Befragten können sich vorstellen, über diese Informationen zu beziehen.

Knapp $40 \%$ der Befragten würden eine App nutzen, die individuell auf deren Bedürfnisse eingeht, während kostenlose Workshops beispielsweise von nur $15 \%$ der Befragten in Anspruch genommen werden würden. $9 \%$ der Befragten würden überhaupt kein Angebot in Anspruch nehmen.

\subsection{Art der gewünschten Information}

Mehr als die Hälfte aller Befragten würden gerne mehr Informationen über die richtige Lagerung bestimmter Lebensmittel erhalten. In weiterer Folge sind Informationen über die möglichen gesundheitlichen Auswirkungen bestimmter Lebensmittel bei deren Verderb ( $47 \%$ der Befragten) sowie Rezepte zur Resteverwertung (44\%) gefragt. $36 \%$ der Befragten haben Interesse an Informationen zur Haltbarmachung von Lebensmitteln. Ein Viertel der Befragten $(26 \%)$ würden gerne erfahren, wie sie sich nach Ablauf des Mindesthaltbarkeitsdatums am besten verhalten können um festzustellen, ob das jeweilige Produkt noch genießbar ist. Nur $16 \%$ der Befragten wünschen sich eine Unterstützung bei der Einkaufs- und Portionsplanung. $10 \%$ der Befragten würde sich keine dieser Informationen wünschen und/oder sie nutzen. Auch in den Kommentaren zeigt sich, dass einige KonsumentInnen empfinden, schon ausreichend oder sogar sehr gut $\mathrm{zu}$ diesen Themen informiert zu sein.

\subsection{Wissensbasis der KonsumentInnen}

Grundkenntnisse im Umgang mit Lebensmitteln sind für die Vermeidung von Lebensmittelabfällen von entscheidender Bedeutung. Es wird davon ausgegangen, dass zusätzlich das Wissen bezüglich Haltbarkeitsdaten, Saisonen und Umweltauswirkungen der Lebensmittelproduktion bzw. von Lebensmit- telabfällen Einfluss auf den Umgang mit diesen haben.

\subsubsection{Haltbarkeitsdaten}

Die falsche Interpretation der Haltbarkeitsdaten - vor allem des Mindesthaltbarkeitsdatums - wird oft als einer der Hauptgründe zum Aufkommen von vermeidbaren Lebensmittelabfällen in Haushalten genannt.

Die Umfrage hat gezeigt, dass KonsumentInnen in Österreich bereits gelernt haben, dass Lebensmittel, die das Haltbarkeitsdatum überschritten haben, verzehrt werden können. $90 \%$ der TeilnehmerInnen würden nach entsprechender Überprüfung von Geruch und Geschmack Milchprodukte essen, die das Haltbarkeitsdatum bereits überschritten haben. Sogar bei Fleisch und Wurstwaren würden $67 \%$ der Befragten die Ware nach Überprüfung noch essen. Bei Reis und Teigwaren geben $45 \%$ der Befragten an überhaupt nicht auf das Haltbarkeitsdatum zu achten.

\subsubsection{Lagerung}

Falsche Lagerung kann die Haltbarkeit von Lebensmitteln und damit das Aufkommen an Lebensmittelabfällen stark beeinflussen. Bei Betrachtung der $\mathrm{Zu}$ sammensetzung von vermeidbaren Lebensmittelabfällen aus österreichischen Haushalten (Schneider und Lebersorger 2009) zeigt sich, dass Obst und Gemüse knapp ein Drittel ausmachen und damit nach Brot und Gebäck am zweithäufigsten entsorgt werden.

Insbesondere die korrekte Lagerung von Obst und Gemüse erfordert Grundkenntnisse über die jeweiligen Merkmale der verschiedenen Sorten, da ein ständiger Kompromiss zwischen der Erhaltung der mikrobiellen und der allgemeinen Qualität gesucht werden muss (Krämer 2011). Beeinflussbare Parameter für die Haushalte sind die Temperatur und die relative Luftfeuchtigkeit.

Bei der vorliegenden Umfrage wie auch einer Masterarbeit mit Lebensmitteltagebüchern (Schwödt 2016) stellte sich heraus, dass besonders Obst häufig fälschlicherweise bei Raumtemperatur gelagert wird, obwohl es von einer kühleren Lagerung profitieren würde. Die Umfrage ergab, dass $58 \%$ der TeilnehmerInnen Weintrauben bei Raumtemperatur lagern, während Äpfel von $78 \%$ bei Raumtemperatur gelagert werden und Orangen sogar von 
$85 \%$ der TeilnehmerInnen. Erdbeeren bleiben nur bei $22 \%$ der TeilnehmerInnen außerhalb des Kühlschranks. Alle aufgeführten Sorten sollten im Kühlschrank aufbewahrt werden, um die Haltbarkeit zu verlängern. Geschmackseinbußen, wie fälschlicherweise oft angenommen, sind nicht zu erwarten, da die Produkte auch davor, bis zu ihrer Präsentation im Einzelhandel (bei Äpfeln z. T. sogar über Monate) entsprechend kühl gelagert werden. $10 \%$ der UmfrageteilnehmerInnen lagern Karotten bei Raumtemperatur, was zu einem deutlich schnelleren Verderb führt. Die optimale Lagerung von Tomaten führt zu merklichen Unsicherheiten bei den KonsumentInnen. Ein österreichischer Tomatenproduzent empfiehlt die Lagerung bei Raumtemperatur. Obwohl Tomaten länger im Kühlschrank frisch bleiben, verlieren sie beträchtlich an Aroma und sind daher nicht für kalte Lagerungsbedingungen geeignet.

\subsubsection{Umweltauswirkungen von Lebensmittelabfall}

Der Wasser-Fußabdruck gibt an, wie viel Wasser für die Produktion bestimmter Lebensmittel benötigt wird. Die UmfrageteilnehmerInnen erhielten eine Auswahl aus sechs verschiedenen Produkten (Reis, Brot, Käse, Rindfleisch, Apfel, Tomate). Die Aufgabe bestand darin, jene drei herauszufinden, die den größten Wasser-Fußabdruck aufweisen. Obwohl nur $41 \%$ der TeilnehmerInnen jene drei mit dem höchsten Wasser-Fußabdruck richtig identifizieren konnten, wussten über $90 \%$, dass die Produktion von Rindfleisch besonders viel Wasser benötigt.

Weniger als $1 \%$ der Befragten ist der Meinung, dass Lebensmittelabfälle kein Problem darstellen, da diese biologisch abbaubar sind. $48 \%$ der Befragten haben bei der Entsorgung von Lebensmitteln Bedenken aufgrund negativer Auswirkungen auf die Umwelt bzw. 45\% aufgrund von negativen Auswirkungen auf die globale Ernährungssituation.

\subsubsection{Erntezeit in Österreich}

Während $77 \%$ der TeilnehmerInnen richtig eingeschätzt haben, dass Kürbisse in Österreich von August bis Oktober geerntet werden können, war es bei anderen Sorten deutlich schwieriger für die Befragten. So wussten nur $28 \%$, dass die Kartoffelernte bereits im Juni beginnt und bis in den Oktober dau- ert, da es sehr unterschiedliche Sorten gibt und nur jene, die im Herbst geerntet werden, auch für die Einlagerung verwendet werden. $50 \%$ der Befragten konnten die heimische Erdbeersaison (Mai bis August) richtig zuordnen.

\subsection{Bereits durchgeführte Vermeidungsmaßnahmen in Haushalten}

\subsubsection{Maßnahmen zur Vermeidung}

Die häufigsten bewusst durchgeführten Maßnahmen, welche von $80-90 \%$ der Befragten regelmäßig genutzt werden, sind das Verwerten von Speiseresten und das Essen oder Verarbeiten von Lebensmitteln, die drohen zu verderben oder das Haltbarkeitsdatum zu überschreiten. Auch Einfrieren spielt eine Rolle für über $80 \%$ der Befragten. Bei Roadshows und Veranstaltungen zum Thema Lebensmittelabfallvermeidung zeigt sich, dass häufig dieselben (wenigen) Lebensmittel bzw. Produkte eingefroren werden und es an Wissen fehlt, welche Bandbreite an Lebensmitteln eingefroren werden können. Die Möglichkeit der Verfütterung an Haustiere, der Weitergabe an Familienmitglieder oder Einkochen wird von etwa $20-30 \%$ der Befragten genutzt. Etwa $6 \%$ der Befragten nutzten bereits die Möglichkeit, Essen an soziale Einrichtungen/ Foodsharing-Initiativen weiterzugeben.

\subsubsection{Einkauf}

Über $80 \%$ der Befragten geben an, immer oder oft zu überprüfen, welche Produkte sich noch im Haushalt befinden, bevor sie einen Einkauf tätigen. In etwa die Hälfte der Befragten gibt an, das Essen für die kommenden Tage immer oder oft im Voraus zu planen, während $8 \%$ der Befragten nur selten und $5 \%$ der Befragten nie eine Einkaufsliste verwenden. Nur $16 \%$ halten sich immer an die Einkaufsliste, weitere $60 \%$ geben an, das oft zu tun.

Aus Sicht der KonsumentInnen führen vor allem Sonderangebote, zu große Verpackungseinheiten oder besonders ansprechende Produkte dazu, dass mehr gekauft wird als ursprünglich geplant. Einkaufen mit Hunger ist ebenfalls für ca. $50 \%$ der Befragten relevant.

\subsubsection{Hindernisse bei der Umsetzung von Vermeidungsmaßnahmen}

Mehr als 55\% der Befragten sind der Meinung, dass mangelnde Zeit, im Haushalt befindliche Lebensmittel noch zu verarbeiten, dazu führt, dass häufiger Lebensmittel entsorgt werden müssen. Ein Viertel der Befragten hat das Gefühl, dass die entsprechende Lagermöglichkeit im Haushalt fehlt. $23 \%$ der Befragten gaben an, dass ihnen die benötigte Kreativität fehlt, um Reste entsprechend zu verwerten. Das fehlende Wissen war für $10 \%$ der Befragten von Bedeutung. $3 \%$ der Befragten gaben an, dass dieses Thema für sie einfach nicht wichtig genug ist.

\section{Erste-Hilfe-Box für Lebensmittel}

Anhand der vorliegenden Ergebnisse wurde ein Maßnahmenpaket, die „Erste-Hilfe-Box“ für Lebensmittel, entwickelt, welches auf die verschiedenen Bedürfnisse der KonsumentInnen abgestimmt wurde. Folgende Punkte wurden bei der Erstellung beachtet:

\subsection{Kommunikation und Kontaktaufnahme}

- Die KonsumentInnen werden nur einmal gezielt mit einem Maßnahmenpaket kontaktiert.

- Das Maßnahmenpaket wird im Einzelhandel angeboten (eine Umsetzung auf Produktebene, wie gewünscht, ist ungleich schwieriger).

- Die von den KonsumentInnen primär gewünschten Informationen (v. a. zu Lagerung, Auswirkungen auf die Gesundheit, Resterezepte und Haltbarmachung) bereitzustellen und zusätzlich darauf zu achten, auch jene Vermeidungsmaßnahmen abzudecken, welche bisher kaum umgesetzt bzw. welche als Hindernisgründe genannt wurden (Zeit, Lagerung, Kreativität), um das Wissen der KonsumentInnen zu vertiefen.

- Verschiedene KonsumentInnengruppen anzusprechen - sowohl jene, denen das Thema bereits sehr wichtig ist und die das Paket als MultiplikatorInnen einsetzen können, als auch jene, die sich bisher wenig mit verschiedenen Maßnahmen beschäftigt haben oder die das Gefühl haben, dass es an Ideen zur Lebensmittelabfallvermeidung mangelt. Ein wesentlicher Punkt war, den KonsumentIn- 


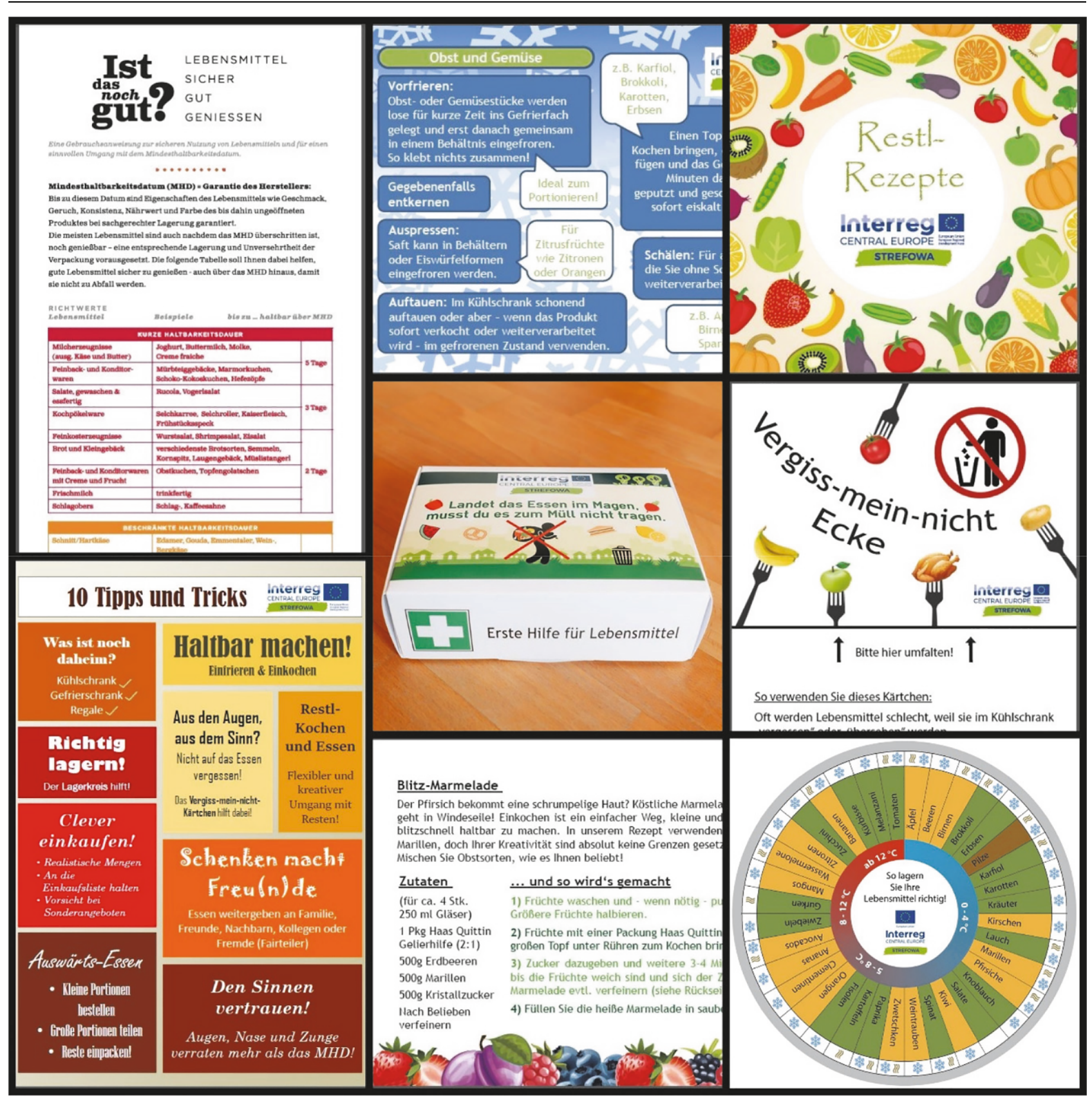

Abb. 1 Erste-Hilfe-Box für Lebensmittel (Außen- und Innenansicht)

nen mehr Sicherheit im Umgang mit Lebensmitteln zu vermitteln.

\subsection{Wissen}

- Die KonsumentInnen werden über die richtige Lagerung von Obst und Gemüse informiert.

- Die KonsumentInnen bekommen Informationen über Haltbarkeitsdaten, wie auch darüber, wie lange Produkte selbst nach Überschreitung dieser
Daten noch genießbar sind, um eine gewisse Sicherheit zu vermitteln.

- Die KonsumentInnen erhalten einen Überblick über mögliche Maßnahmen im Haushalt.

\subsection{Handeln}

- Die KonsumentInnen bekommen kreative Resterezepte zur Verfügung gestellt, deren Erarbeitung von FoodbloggerInnen unterstützt wurde.
- Die KonsumentInnen erhalten Informationen zum Einfrieren sowie Gefrierbeutel zum Handeln.

- Die KonsumentInnen erhalten Informationen zum Einkochen sowie Gelierhilfe zum Handeln.

- Die KonsumentInnen erhalten eine Hilfestellung, die verhindern soll, dass Lebensmittel im Kühlschrank vergessen werden.

Abb. 1 zeigt die Außenansicht sowie die entwickelten Inhalte für die „Erste-Hil- 
fe-Box“, welche in weiterer Folge im Detail beschrieben werden.

Für das Design der Box wurde einerseits das bekannte Zeichen für „Erste Hilfe" an der Frontseite verwendet und andererseits eine Abbildung einer durchgestrichenen Figur, die Lebensmittel zum Müll trägt, für die Vorderseite gewählt, zusammen mit dem Spruch „Landet das Essen im Magen, musst du es zum Müll nicht tragen“, der an eine Bauernregel erinnert, wie sie in Österreich bzw. allgemein im deutschsprachigen Raum oft verwendet werden, um das Wetter vorherzusagen.

Folgende Informationsmaterialien wurden für die Erste-Hilfe-Box entwickelt:

- 10 Tipps und Tricks: Dieses Informationsblatt im A5-Format zeigt einen Überblick über Lebensmittelabfallvermeidungsmaßnahmen im Haushalt.

- Blitz-Marmelade: Einkochen ist unkompliziert und geht schnell - dies ist eine wichtige Botschaft an KonsumentInnen, die befürchten zu wenig Zeit dafür zu haben, um Lebensmittel auf diese Weise haltbar zu machen. In vier einfachen Schritten wird auf dem BlitzmarmeladeKärtchen erklärt, wie Marmelade eingekocht werden kann, um damit Obst haltbar zu machen, zusätzlich finden sich wertvolle Tipps, wie die Marmelade verfeinert werden kann und worauf es beim Einfüllen in die Gläser ankommt

- Lagerkreis: Der Lagerkreis hilft KonsumentInnen dabei, Obst und Gemüse richtig zu lagern. Er zeigt, welche Temperaturen für die Lagerung bestimmter Obst- und Gemüsesorten geeignet sind, welche eingefroren werden können und welche große Mengen des Reifegases Ethylen abgeben.

- Vergiss-Mein-Nicht-Ecke: Mit dieser kann ein bestimmter Bereich im Kühlschrank markiert werden, in dem in weiterer Folge Lebensmittel gelagert werden, die zeitnah gegessen werden müssen. Dies soll KonsumentInnen dabei unterstützen, nicht auf schnell verderbliches Essen im Kühlschrank zu vergessen.

- Einfrier-Hilfe: Einfrieren wird bereits von vielen KonsumentInnen genutzt. Das Einfrierkärtchen bietet einen detaillierten Überblick, welche Lebensmittel wie lange eingefroren werden können. Dabei spielt auch das schonende Auftauen eine Rolle.
Folgende Materialien wurden außerdem zur Verfügung gestellt:

- Toppits-Gefrierbeutel: Damit es den KonsumentInnen leichter fällt, die Einfriertipps in die Tat umzusetzen, wurden von der Firma Toppits Gefrierbeutel gesponsert.

- Haas-Gelierhilfe: Die von der Firma Haas gesponserte Gelierhilfe soll KonsumentInnen dazu ermuntern, Obst mithilfe des Blitzmarmeladekärtchens einzukochen.

- „Ist das noch gut?"-Folder der Wiener Tafel: Dieser Folder erklärt das Mindesthaltbarkeitsdatum und zeigt, wie lange verschiedene Produkte oft noch nach Überschreiten des Datums gegessen werden können, um KonsumentInnen diesbezüglich mehr Sicherheit zu geben.

\section{Evaluation der Erste-Hilfe-Box}

Die Evaluation von Vermeidungsmaßnahmen wird nach wie vor meist nicht durchgeführt. Daher ist es oft nicht möglich abzuschätzen, inwiefern diese auch von den KonsumentInnen angenommen wurden.

\subsection{Methode}

Die Erste-Hilfe-Boxen wurden im März 2018 innerhalb von 6 Tagen in drei verschiedenen SPAR-Filialen in Wien an 2000 VerbraucherInnen verteilt. Die Filialen befinden sich in verschiedenen Wiener Gemeindebezirken, die sich auch bezüglich Einkommens-, und Bildungsniveau und Arbeitslosenanteil voneinander unterscheiden. Dies sollte es ermöglichen, mit der Box möglichst viele unterschiedliche Typen von KonsumentInnen $\mathrm{zu}$ erreichen. Die Verteilung selbst fand $\mathrm{zu}$ verschiedenen Tageszeiten unter der Woche sowie am Wochenende statt, was ebenso die Erreichung möglichst unterschiedlicher KonsumentInnengruppen ermöglichte.

Die Evaluierung der Erste-Hilfe-Box erfolgte wiederum über eine Befragung der KonsumentInnen, indem der Box jeweils ein Kuvert mit Antwortsendung beigelegt wurde. Die Antwortsendung ermöglicht es, dass der Empfänger den Versand übernimmt, und zwar nur für die Kuverts, die tatsächlich zurückgeschickt werden. Den KonsumentInnen wurden die Boxen persönlich ausgehändigt und die weitere Vorgehensweise sowie das Ziel dahinter kurz erklärt. Zusätzlich wurde es den KonsumentInnen ermöglicht, den Fragebogen online auszufüllen - der dafür benötigte Link war ebenfalls in der Box zu finden. Um die Rücklaufquote zu erhöhen, wurden Preise zur Verfügung gestellt, die unter all jenen verlost wurden, die einen Fragebogen retournierten und damit einverstanden waren, ihre Kontaktdaten weiterzugeben.

307 KonsumentInnen retournierten einen Fragebogen. Das bedeutet, dass $15 \%$ der Haushalte, die eine Box erhielten, an der Befragung teilnahmen. In der Regel arbeiten Marktforschungsinstitute mit Follow-Ups und Erinnerungen - sie wenden sich somit erneut an die zu befragenden Personen um die Rücklaufquote zu erhöhen. Dies war jedoch mit dieser Art der Verteilung nicht möglich - die Rücklaufquote kann als zufriedenstellend angesehen werden.

\subsection{Ergebnisse}

Da diese Stichprobe nicht selbst ausgewählt wurde, sondern vollständig auf freiwilliger Teilnahme beruhte, war die Zusammensetzung nicht vorhersehbar. Die Mehrheit der TeilnehmerInnen war weiblich $(77,5 \%)$ und das Durchschnittsalter der TeilnehmerInnen betrug 52,7 Jahre, was deutlich über dem österreichischen Durchschnittsalter von 42,6 Jahren zu Beginn des Jahres 2018 liegt (Statistik Austria 2018). 45\% der TeilnehmerInnen waren zum Zeitpunkt der Befragung unter 50 Jahren alt. KonsumentInnen zwischen 15 und 80 Jahren konnten zur Teilnahme an der Evaluierung motiviert werden.

Im Durchschnitt lebten die TeilnehmerInnen in einem 2,3-PersonenHaushalt, was annähernd mit dem österreichischen Durchschnitt von 2,15 vergleichbar ist (Statistik Austria 2017). $21,8 \%$ der TeilnehmerInnen gaben an, in ihrem Haushalt mit Kindern unter 18 Jahren zu leben. Die teilnehmenden Haushalte haben durchschnittlich 1,8 Kinder, was in etwa dem österreichischen Durchschnitt von 1,67 entspricht (Statistik Austria 2017). 24\% der TeilnehmerInnen gaben an, ein Haustier zu besitzen.

Eine große Mehrheit (76,8\%) der TeilnehmerInnen gab an, extrem betroffen davon $\mathrm{zu}$ sein, Lebensmittel entsorgen zu müssen, weitere $21 \%$ empfinden es als „ziemlich unangenehm“. Nur ein/e Teilnehmer/in gab an, dass das Wegwerfen von Essen nicht als unangenehm empfunden wird. 
Hier steht eine Anzeige.

Springer 
Hier steht eine Anzeige.

Springer 


\section{In jeder Box finden sich Helfer zu unterschiedlichen Themen. Welche drei würden Sie am ehesten im Alltag einsetzen? $(\mathrm{N}=307)$}

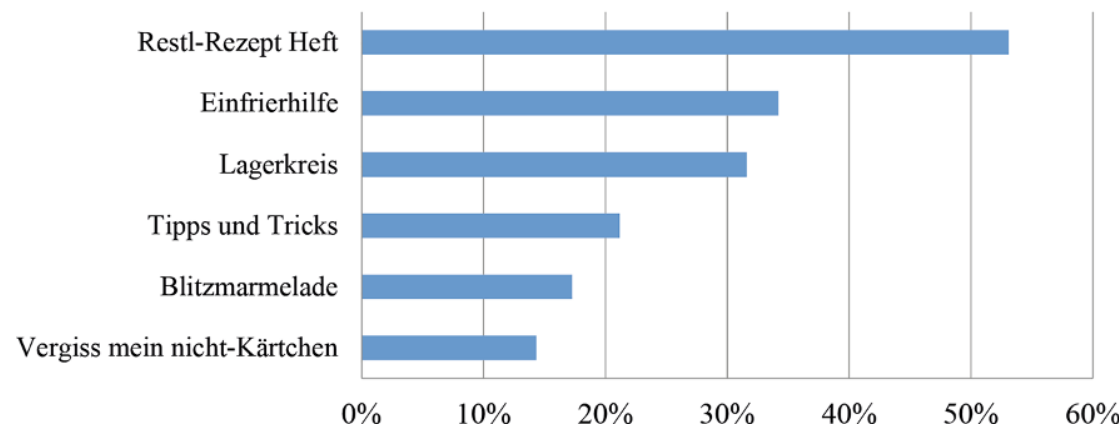

Abb. 2 Alltagstauglichkeit der Materialien aus Sicht der KonsumentInnen

\section{Welche der folgenden Aussagen treffen auf Sie zu?} $(\mathrm{N}=307)$

Ich gehe achtsamer mit Lebensmitteln um.

Ich werfe nun weniger weg.

Ich werde die Inhalte wieder nutzen.

Die Inhalte sind interessant

Ich habe etwas Neues gelernt.

Das Thema nervt mich.

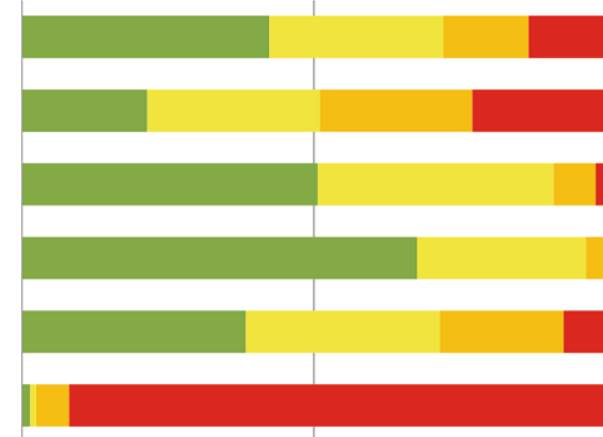

$0 \%$

$50 \%$

$100 \%$

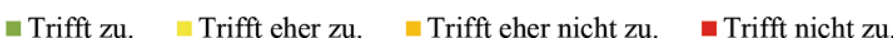

Abb. 3 Meinungen der KonsumentInnen zu verschiedenen Aussagen zur Box

Abb. 2 zeigt, welche drei Materialien aus der Box KonsumentInnen am ehesten im Alltag einsetzen würden.

Das beliebteste entwickelte Material stellt das Rezeptheft dar, welches mehr als $50 \%$ wiederverwenden würden. Auch das Einfrierkärtchen sowie der Lagerkreis wurden von mehr als $30 \%$ als nützlich bewertet. Die 10 Tipps zur Lebensmittelabfallvermeidung wurden von $21 \%$ der TeilnehmerInnen geschätzt, während das „Vergiss-meinnicht-Kärtchen“ nur wenige begeistern konnte.

Unter den gesponserten Materialien sind die „Toppits-Gefrierbeutel“ am beliebtesten - $65 \%$ der TeilnehmerInnen schätzen dieses Hilfsmittel. Etwa $20 \%$ der Konsumenten empfanden die „Haas-Gelierhilfe“ als hilfreich. Der „Ist das noch gut?"-Folder der Wiener Tafel stieß bei $44 \%$ der TeilnehmerInnen auf Interesse.
Abb. 3 zeigt, inwiefern verschiedene Aussagen zu der Box auf die TeilnehmerInnen zutreffen.

Über $90 \%$ der TeilnehmerInnen gaben an, dass sie nicht oder eher nicht behaupten würden, von dem Thema „Lebensmittelabfall“ genervt zu sein. Mehr als $65 \%$ der Teilnehmer empfinden den Inhalt als interessant, für weitere $28 \%$ ist das eher zutreffend. $36 \%$ der Befragten empfinden es als zutreffend, etwas Neues gelernt zu haben, für weitere $31 \%$ ist diese Aussage eher zutreffend. Laut Aussagen der TeilnehmerInnen hat die Erste-Hilfe-Box auch Einfluss auf deren Verhalten. So würden $46 \%$ der TeilnehmerInnen die zu bewertende Aussage „Ich werfe nun weniger weg" als zutreffend oder eher zutreffend empfinden. $40 \%$ der Befragten empfinden es als zutreffend und weitere $28 \%$ als eher zutreffend, nun schonender mit Lebensmitteln umzugehen. Für beinahe $90 \%$ der Teilneh-
merInnen ist die Aussage „Ich werde den Inhalt wieder verwenden" zutreffend oder eher zutreffend.

Zusätzlich wurden die Teilnehmer gefragt, ob sie sich andere nützliche Hilfestellungen vorstellen können, die durch die Erste-Hilfe Box oder ähnliche Materialien zukünftig aufgegriffen werden können.

$24 \%$ der TeilnehmerInnen, die gerne ihre Idee teilen wollten, gaben an, dass sie eine größere Vielfalt an Resterezepten vorziehen würden. Darüber hinaus haben $22 \%$ der TeilnehmerInnen Interesse an einer Liste mit sozialen Einrichtungen, an welche Lebensmittelüberschuss aus Haushalten übergeben werden kann. Vereinzelt werden mehr Informationen zu Haltbarkeitsdaten gewünscht oder aber z. B. Listen, die am Gefrierschrank angebracht werden können, um einen Überblick über die eingefrorenen Lebensmittel $\mathrm{zu}$ behalten, bzw. Magnete, um die Vergissmein-nicht-Kärtchen zu ersetzen oder aber die unterschiedlichen Temperaturzonen im Kühlschrank zu markieren. In weiterer Folge gab es Anmerkungen da$\mathrm{zu}$, welche Lebensmittel als Tierfutter für Haus- und/oder Wildtiere geeignet sind, und eine Liste an Abfallvermeidungs-Apps für KonsumentInnen. Weitere 30 TeilnehmerInnen gaben an, keine andere Idee zu haben oder nichts ändern zu wollen.

Die Ergebnisse zeigen, dass bei der Entwicklung von Vermeidungsmaßnahmen auch sozio-demografische Merkmale eine Rolle spielen. Die Gefrierhilfe, der Lagerkreis, aber auch die Vergissmein-nicht-Kärtchen werden von den unter 35-Jährigen am besten bewertet, während z.B. die Blitzmarmelade bei dieser Altersgruppe am wenigsten Anklang fand. Die Tipps und Tricks bzw. der „Ist das noch gut?“-Folder der Wiener Tafel konnte bei TeilnehmerInnen zwischen 35 und 65 Jahren punkten. Die Resterezepte waren bei über $50 \%$ der Teilnehmer alle Altersgruppen beliebt, überraschenderweise jedoch besonders bei den über 65-Jährigen.

Auch das Geschlecht hat einen gewissen Einfluss bezüglich der Präferenz bestimmter Materialien. Die Einfrierkärtchen, die „Ist das noch gut?“-Folder sowie der Lagerkreis werden von Männern mehr geschätzt als von Frauen. Im Gegensatz dazu gefallen Frauen die Rezepte sowie das Blitzmarmeladekärtchen bedeutend besser.

Auch der Bildungsgrad hat einen Einfluss auf die Beliebtheit bestimm- 
ter Box-Inhalte. Der Lagerkreis wurde deutlich besser (mehr als doppelt so gut) von TeilnehmerInnen mit Matura oder abgeschlossenem Studium bewertet als von TeilnehmerInnen, deren höchste abgeschlossene Ausbildung eine berufsbildende Schule oder Lehre ist. Das Marmeladerezept sowie die 10 Tipps und Tricks werden von AbgängerInnen von berufsbildenden Schulen am meisten geschätzt, gleichzeitig bewertet diese Gruppe die Einfrierhilfe am schlechtesten.

\section{Diskussion und Schlussfolgerungen}

Lebensmittelverschwendung ist ein Thema, das die KonsumentInnen bewegt - doch reicht diese Betroffenheit beim Blick in die Tonne aus, um das Aufkommen von Lebensmittelabfällen in Haushalten nachhaltig zu verringern? Wie können jene KonsumentInnen erreicht werden, die sich überhaupt nicht dafür interessieren? Die Absicht Lebensmittelabfälle $\mathrm{zu}$ vermeiden, führt jedenfalls nicht zwangsläufig zu einer tatsächlichen Reduktion dieser Abfälle (Stefan et al. 2013). Wichtig erscheint daher in einem ersten Schritt auf die passenden Maßnahmen zu finden.

Auf bestimmte Konsumententypen angepasste Maßnahmen können ein dabei ein Schlüssel zum Erfolg sein. Die Auswertung der Erste-Hilfe-Box zeigt, dass auch die Berücksichtigung sozio-demografischer Merkmale wie z.B. Alter oder Bildungsgrad sinnvoll ist. Auch Emotionen sind ein wichtiger Faktor - Schuldgefühle beispielsweise können die Motivation zum Handeln erhöhen (Quested et al. 2013). Auch die Erste-Hilfe-Box wurde in erster Linie von KonsumentInnen evaluiert, welche es als sehr unangenehm empfinden, Lebensmittel zu entsorgen.

Ein weiterer wichtiger Punkt ist die Kommunikation von Lebensmittelabfallvermeidung - es scheint sinnvoll zu sein, eine Mischung aus verschiedenen Medien bei Kampagnen einzusetzen. Die vorliegenden Ergebnisse zeigen, dass der Einzelhandel bei der Verbreitung von Vermeidungsmaßnahmen aus Sicht der KonsumentInnen eine wichtige Rolle spielt, was auch in der Studie von Young et al. (2017) deutlich wird. Diese zeigt, dass durch die Vermittlung entsprechender Inhalte auf SocialMedia-Kanälen von Einzelhandelsunternehmen bzw. in Newslettern und Magazinbeiträgen des untersuchten
Einzelhandelsunternehmens sowohl die Häufigkeit als auch die Mengen der gemeldeten Lebensmittelabfälle reduziert werden konnten.

Die Erste-Hilfe-Box ist ein Beispiel für eine innovative, an die Bedürfnisse der KonsumentInnen angepasste Maßnahme, mit deren Hilfe es jedenfalls möglich, ist die Neugier der KonsumentInnen $\mathrm{zu}$ wecken, um herauszufinden, was sich im Inneren befindet - einen ersten Kontakt herzustellen ist ein wichtiger Schritt. Obwohl sich gezeigt hat, dass KonsumentInnen es bevorzugen, nur einmalig kontaktiert zu werden, um zu erfahren, wo sich die entsprechenden Informationen auffinden lassen, sind regelmäßige Erinnerungen sicher sinnvoll.

Obwohl die KonsumentInnen bei der Befragung zur Erste-Hilfe-Box feststellten, nun weniger wegzuwerfen, die Materialien wiederzuverwenden oder sorgsamer mit Lebensmitteln umzugehen, ist ungewiss, ob sich das Aufkommen an Lebensmittelabfällen in diesen Haushalten tatsächlich verändert hat. Die Art der Evaluierung lässt keinen direkten Rückschluss darauf zu, ob Personen ihr Handeln tatsächlich ändern, sondern nur auf die Einstellung der Menschen zur Box. Die Erhebung von Effekten von Abfallvermeidungsmaßnahmen generell wird von ExpertInnen als schwierig eingeschätzt (z.B.: Zorpas und Lasaridi 2013; Read et al. 2009). Zentrale Probleme, die sich hier ergeben, sind zum einen, dass man Vermeidungsmaßnahmen nicht sehen kann und eine Teilnahme daran visuell nicht beobachtbar ist, im Gegensatz zu Recyclingmaßnahmen. Zum anderen ist es schwierig, eine Reduktion der Abfallmenge der Vermeidung zuzuschreiben und nicht etwa anderen Umständen, wie der wirtschaftlichen Situation. Gängige Erhebungsmethoden zu Effekten der Abfallvermeidung sind u.a. Analysen zum Abfallaufkommen, vergleichende Analysen mittels Kontrollgruppen, Analysen mittels spezifischer zumeist räumlich abgegrenzter Kampagnen, Analysen von Verhaltensänderungen mittels Befragungen, teilnehmende Beobachtungen und andere. All diese Methoden haben Stärken aber auch Schwächen. Sehr oft sind die Methoden zu aufwendig, zu personalund kostenintensiv, um sie ausführlich genug für repräsentative Ergebnisse zu gestalten. Unbestritten besteht auf diesem Gebiet weiterer Forschungsbedarf.
Vermeidungsmaßnahmen werden in Zukunft möglicherweise nicht nur darauf fokussieren, die Menge an vermeidbaren Lebensmittelabfällen zu reduzieren, sondern sich auch verstärkt auf Lebensmittel mit den relevantesten Umweltauswirkungen und umweltbewusste Kaufentscheidungen konzentrieren. Young et al. (2017) kommen zum Schluss, dass es wichtig ist, bei der Entwicklung von Lebensmittelabfallvermeidungsmaßnahmen in Hinblick auf die Vermeidung von Treibhausgasemissionen bzw. andere negative Umweltauswirkungen auch darauf $\mathrm{zu}$ achten, sogenannte Rebound-Effekte zu vermeiden. Diese können dann entstehen, wenn KonsumentInnen z. B. das durch Vermeidung von Lebensmittelabfällen mit geringerer Umweltauswirkung (z. B. Obst, Gemüse) gesparte Geld dazu verwenden, Produkte zu kaufen, deren Produktion mit großen Mengen an Treibhausgasemissionen verbunden ist, wie z. B. Fleisch (Barrett und Scott 2012).

Diese Überlegungen sprechen dafür, dass es in erster Linie von Bedeutung ist, die Wertschätzung für Lebensmittel entlang der gesamten Wertschöpfungskette zu erhöhen - dazu werden in den kommenden Jahren noch mehr Anstrengungen erforderlich sein und kreative Wege eingeschlagen werden müssen. Nur dann wird es gelingen, das Entwicklungsziel 12 der Vereinten Nationen „Nachhaltiger Konsum und Produktion" auch umzusetzen und wie in 12.3 gefordert auch die Menge an Lebensmittelabfällen pro Kopf bis 2030 zu halbieren.

Förderung Das Projekt STREFOWA wurde finanziert durch die Mittel des Europäischen Development Fonds und kofinanziert durch das Bundesministerium für Nachhaltigkeit und Tourismus, die Wiener Umweltschutzabteilung (MA 22) sowie der VKS Verpackungskoordinierungsstelle $\mathrm{GmbH}$.

Funding Open access funding provided by University of Natural Resources and Life Sciences Vienna (BOKU). 


\section{Originalarbeit}

Open Access Dieser Artikel wird unter der Creative Commons Namensnennung 4.0 International Lizenz (http:// creativecommons.org/licenses/by/4. 0/deed.de) veröffentlicht, welche die Nutzung, Vervielfältigung, Bearbeitung, Verbreitung und Wiedergabe in jeg- lichem Medium und Format erlaubt, sofern Sie den/die ursprünglichen $\mathrm{Au}$ tor(en) und die Quelle ordnungsgemäß nennen, einen Link zur Creative Commons Lizenz beifügen und angeben, ob Änderungen vorgenommen wurden.
Hinweis des Verlags Der Verlag bleibt in Hinblick auf geografische Zuordnungen und Gebietsbezeichnungen in veröffentlichten Karten und Institutsadressen neutral.

\section{Literatur}

Barrett, J., Scott, K, (2012): Link between climate change mitigation and resource efficiency: a UK case study. Global Environ. Change 22, 299-307.

Krämer, J. (2011): Lebensmittel-Mikrobiologie. Ulmer-Verlag: 6. Auflage, Bonn: 131-213

Obersteiner, G., Schwödt, S. (2017): Report on existing barriers and future concepts on food waste prevention at households. STREFOWAReport.

Quested, T., Marsh, E., Stunell, D., Parry, A. (2013): Spaghetti soup: the complex world of food waste behaviours Resour. Conserv. Recycl., 79 (2013), pp. 43-51

Read, M., Gregory, M., Phillips, P. (2009): An evaluation of four key methods for monitoring household waste prevention campaigns in the UK. Resources, Conservation \& Recycling, 2009, Band 54, Ausgabe 1, pp. 9-20

Schneider, F., Lebersorger, S. (2009): Untersuchung der Lebensmittel im Restmüll in einer oberösterreichischen Region. Projektbericht im Auftrag des Amts der OÖ Landesregierung, Abteilungen Umweltschutz und Land- und Forstwirtschaft. Verfügbar unter: http://www. landoberoesterreich.gv.at/files/publikationen/ US_lebensmittel_restmuell.pdf (11.03.2012)

Schwödt, S. (2016): Einfluss des Vertriebsweges auf das Aufkommen von Obst- und Gemüseabfällen beim Konsumenten. Masterarbeit - Institut für Abfallwirtschaft (ABF-BOKU), BOKUUniversität für Bodenkultur

Statistik Austria (2017): Privathaushalte nach Geburtsland der Haushaltsreferenzperson, Haushaltsgröße und Bundesländern http://www. statistik.at/web_de/statistiken/menschen_und_ gesellschaft/bevoelkerung/haushalte_familien lebensformen/haushalte/023303.html (29.5.2018) Statistik Austria (2018): Bevölkerung nach Alter und Geschlecht. https://www.statistik.at/web_ de/statistiken/menschen_und_gesellschaft/ bevoelkerung/bevoelkerungsstruktur/ bevoelkerung_nach_alter_geschlecht/index.htm (29.05.2018)

Stefan, V, Van Herpen, E, Tudoran, A. A, Lähteenmäki, L. (2013): Avoiding food waste by Romanian consumers: the importance of planning and shopping routines. Food Qual. Prefer., 28 (2013) pp. 375-381

Stenmarck, A., Jensen, C., Quested, T., Moates, G. (2016): Estimates of European Food Waste Levels. IVL Swedish Environmental Research Institute. Sweden.

Young, W., Russell, S.., Robinson, C., Barkemeyer, R. (2017): Can social media be a tool for reducing consumers' food waste? A behaviour change experiment by a UK retailer. Resour. Conserv. Recycl., 117 (2017) 195-203

Zorpas, A., Lasaridi, K. (2013): Measuring waste prevention. Waste Management, 05/2013, Band 33, Ausgabe 5, pp. 1047-1056 